Volume 8, No. 7, July - August 2017

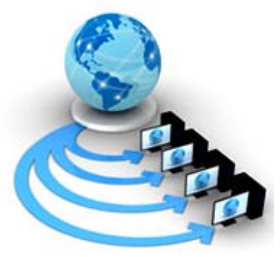

International Journal of Advanced Research in Computer Science

RESEARCH PAPER

\author{
Available Online at www.ijarcs.info
}

\title{
ANALYZING THE EFFECTS OF MEDITATION ON ELECTROENCEPHALOGRAPH SIGNALS
}

\author{
Kulvir Kaur \\ Department of Electronics and Communication Engineering \\ Baba Banda Singh Bahadur Engineering College \\ Fatehgarh Sahib, Punjab, India
}

\author{
Karamjeet Singh \\ Department of Electronics and Communication Engineering \\ Baba Banda Singh Bahadur Engineering College \\ Fatehgarh Sahib, Punjab, India
}

\author{
R.S.Uppal \\ Department of Electronics and Communication Engineering \\ Baba Banda Singh Bahadur Engineering College \\ Fatehgarh Sahib, Punjab, India
}

\begin{abstract}
People are adopting various meditation techniques to make themselves comfortable in their daily routines. These practices which came from rich traditions are investigated in scientific framework. This paper examined the effects of meditation on human brain. These scientific investigations on meditation practices have attracted a lot of researchers across the globe. In this research human behaviour is recorded, examined and investigated before and after meditation intervention. This paper describes the influence of meditation on the brain signals to improve mental health. In this paper Nexus machine was used for recording of data and LabVIEW (Laboratory Virtual Instrument Engineering Workbench) and SPSS (Statistical Package for Social Sciences) software tools were used to implement the project.
\end{abstract}

Keywords: Electroencephalography, meditation, brain signals, SPSS, LabVIEW.

\section{INTRODUCTION}

A lot of research has been undertaken in assessment of brain functionality over the years. Human brain is the most significant and complex organ of the human body through which a person is able to respond to various sensory stimuli as well as perform complex activities [1]. The brain is the supreme commander of the human body. It is the central part of the nervous system which governs the various activities such as cognition, emotions, language, sleep, wakefulness, and memory are made possible by the brain. There are too many aspects of the human brain that still remain unexplored. Understanding the behavior of the brain is an interesting area for researchers to find out better solution for various brain related disorders [2].There are many neurophysiologic techniques such as Computed Tomography (CT), Positron Emission Tomography (PET) and Magnetic Resonance Imaging (MRI) can be used in diagnosing brain related abnormalities [3,4,5].

Electroencephalography (EEG) is the most popular and efficient technique which is an inexpensive and a relatively simple method to study the activity of the brain. Most significant characteristics of EEG incorporates non invasive, safe, pain less and precise interpretations for diagnosis of various brain related diseases such as, sleep disorders, epilepsy, Alzheimer, depression, Parkinson, sclerosis, coma, brain death autism[6,7,8].EEG provides high temporal resolution of the signals by recording the fluctuations of electrical potentials. Moreover, EEG detects changes within a millisecond timeframe [9]. This technique has been used effectively in cognitive research over decades.
Meditation and its effect on brain activity became a vital area for research in neuroscience, psychology and neurobiology. Research on meditation defines various benefits and changes in brain waves. Modern lifestyles characterized by a significant amount of mental stress owing to hectic work schedules, often leads to negative emotions, depression, insomnia and other symptoms after an extended period. Previous studies showed that meditation can significantly affect physical and mental relaxation [10-13]. Meditation is a type of complementary medicine treatment.

This paper presents EEG based study to analyze the brain waves of the subject. Section II describes methods of recording and processing EEG signals. Results and their discussion are explained in the section III. Section IV concludes the paper.

\section{METHODOLOGY}

\section{A. Participants and training}

The EEG signals were obtained from 25 healthy volunteers (16males and 9 females; aged 20-47) without any previous meditation training experience. All the subjects were given meditation training, included a 60-minute session daily for four weeks, by a trained and experienced teacher. Meditation intervention included a body-awareness meditation. 


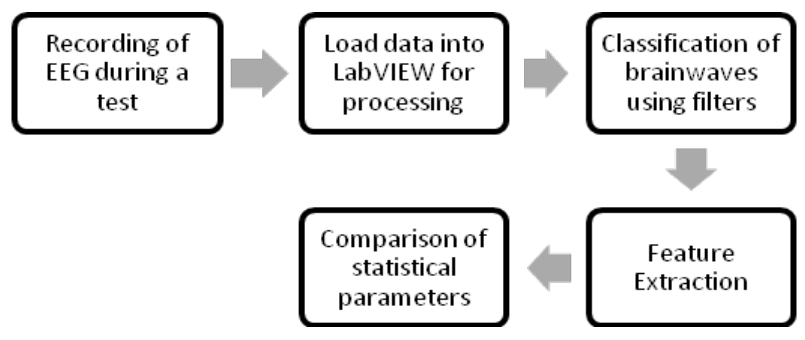

Figure 1. Block Diagram of the process

\section{B. EEG Recording}

The EEG signals were recorded during a test for 10 minutes and after four week meditation session; signals were recorded once again during test of equal difficulty. During pretest, scores of all the participants were saved for comparison with post-test results.

The data was collected using 8 EEG channels: F3, F4, C3, C4, P3, P4, O1 and O2 with reference and ground electrodes. The international 10-20 method was used for electrode placement. The hardware used for data acquisition was Nexus machine. The data was recorded at a sampling rate of $256 \mathrm{~Hz}$ by standard surface silver/silver chloride (Ag/AgCl) scalp electrodes. The Fig. 1 shows one of the recorded EEG signal.

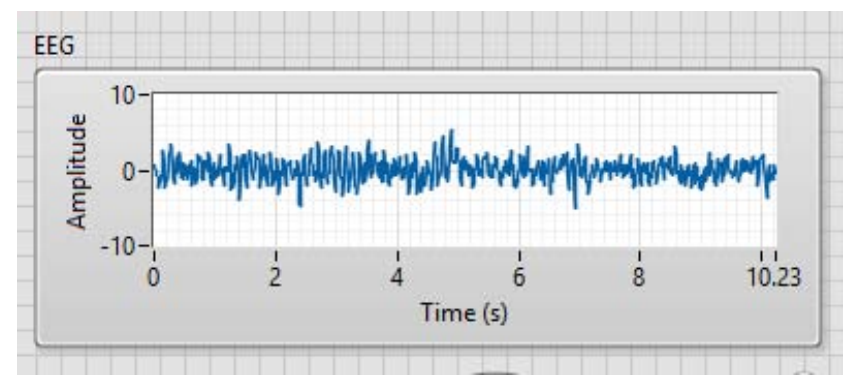

Figure 2. EEG signal

\section{EEG Processing}

After acquiring the EEG signals via the electrodes, differential amplifiers were used to amplify these signals and then a filter block was used to reduce the noise frequencies and obtain smooth signals.

LabVIEW software was used for signal filtering and feature extraction. The full form of the LabVIEW is (Laboratory Virtual Instrument engineering workbench) is invented for environment based on the graphical programming. The lab view is used terminology; icons and ideas are understandable to technician, scientist and engineers and it relies on graphical symbols rather than textual language to run the programs. It is graphical programming that uses icons instead of lines of text to create applications

The signals acquired were filtered with the help of LabVIEW software and alpha, beta, delta, theta and gamma frequency band were extracted from it. Various filters were available in LabVIEW which can be used to filter EEG signals. In this study, IIR filters were preferred over FIR filters because of faster and more efficient filtering with non linearphase response. Thus, The EEG signals were filtered to obtain different frequency rhythms using IIR Butterworth band pass filter to keep flat response and desired sharper cut off. Five filters for five different bands were used with the same filter characteristics. In Fig. 2, the block diagram of signal processing is shown.

A band pass filter with pass band frequency .5Hz and stop band frequency $3 \mathrm{~Hz}$ was used to obtain Delta frequency bands. Similarly, by using a band pass filter with pass band frequency $4 \mathrm{~Hz}$ and stop band frequency of $7 \mathrm{~Hz}$, Theta waves were obtained. Alpha waves were obtained using band pass filter with pass band frequency $8 \mathrm{~Hz}$ and stop band frequency $12 \mathrm{~Hz}$. Similarly, a band pass filter with pass band frequency $13 \mathrm{~Hz}$ and stop band frequency $30 \mathrm{~Hz}$ was used to obtain Beta frequency band. For gamma waves, a band pass filter with pass band frequency $35 \mathrm{~Hz}$ and stop frequency $100 \mathrm{~Hz}$ was used. Further, the corresponding waveforms were displayed using waveform graphs are shown in Fig. 2.

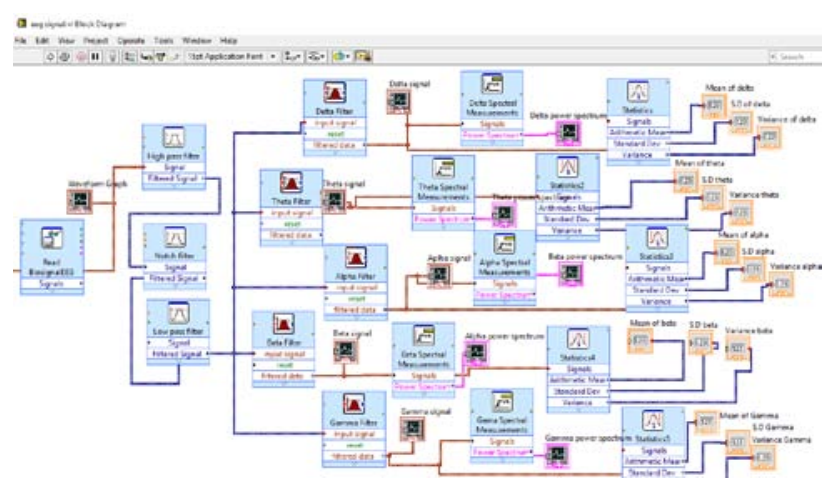

Figure 3. Block diagram of signal processing in LabVIEW

\section{Feature extraction}

In this paper, the mean, entropy, standard deviation and variance were calculated from time analysis and FFT was used for spectral analysis.

- $\quad$ FFT: A fast Fourier transform (FFT) is an important and efficient tool for the feature extraction. FFT is performed on the data in order to see it in the frequency domain. Fourier analysis converts a signal from time domain to a representation in the frequency domain and vice versa.

The FFT is can compute the result $\mathrm{O}(\mathrm{N} \log \mathrm{N})$ operation, where $\mathrm{N}$ is the length of the vector. There will be the range of the $\mathrm{N}$ in thousand or million so that the calculation is very complex and time consuming. Thus to reduce the operation time and increasing the speed by FFT is preferred over DWT.

- $\quad$ Standard Deviation: Standard Deviation is defined as the how much difference or "dispersion" exists from the average (mean, or expected value). A low standard deviation specifies the data points tend to be very close to the mean; high standard deviation indicates that the data points are spread out over a large range of values.

$$
\operatorname{S.D}(\sigma)=\frac{\sqrt{\sum(\mathrm{x}-\mu)}}{\mathrm{N}}
$$

Where, $\sigma=$ Symbol of Standard Deviation $\mu=$ mean of all the values in the data set $\mathrm{N}=$ Total Number of values in data set $\mathrm{x}$ $\mathrm{x}=$ each value in the data set $\mathrm{D}$

- Variance: Variance is defined as the square of standard deviation or the variance of a random variable or distribution is the probability, or mean, of 
the squared deviation of that variable from its estimated value or mean. Variance is a measure of dispersion of the data. Thus the variance is a degree of measure of the amount of variation in values of that variable, taking account of all probable values and their probabilities.

- $\quad$ Entropy: Entropy is a measure of signal randomness and information content of the signal.

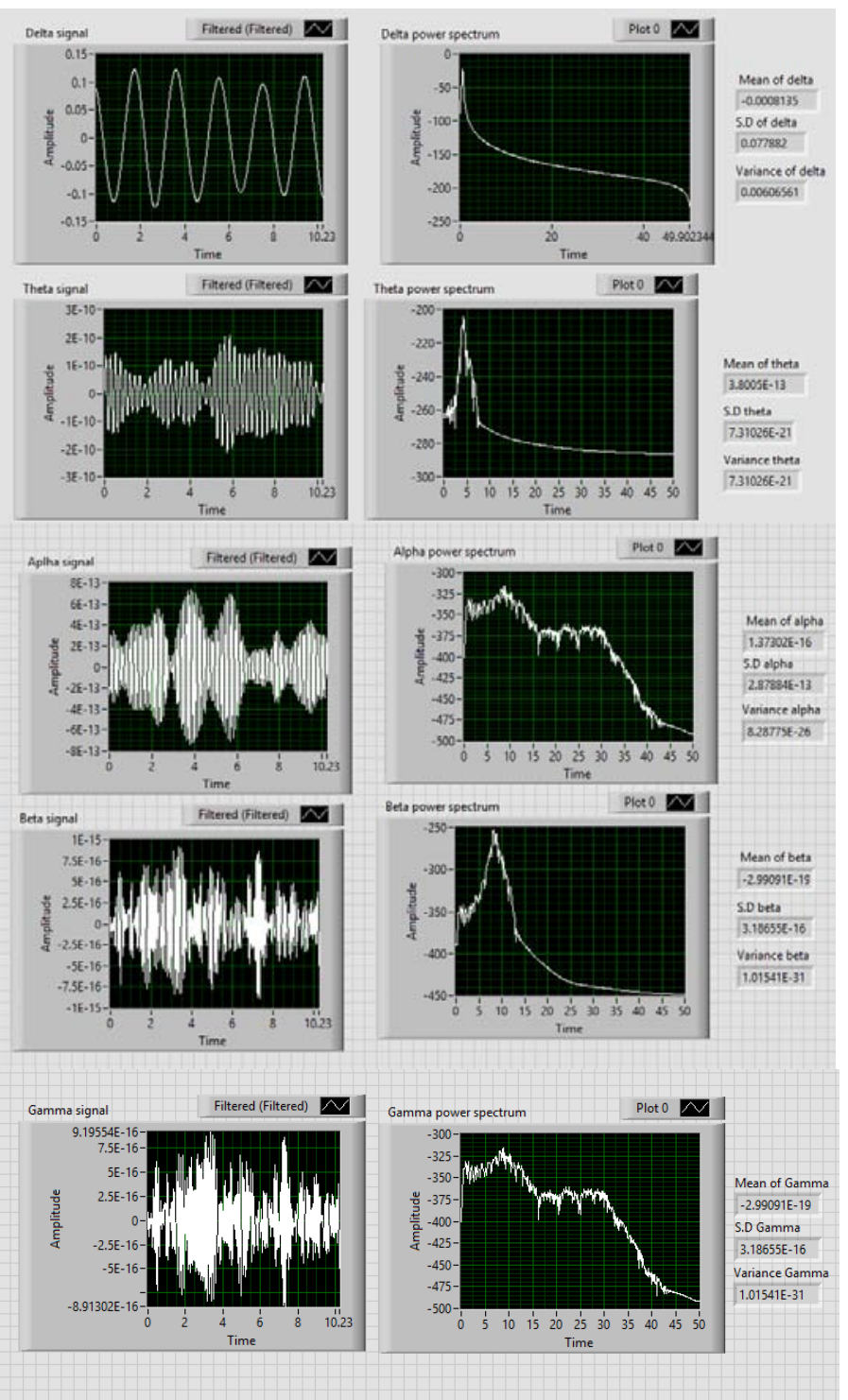

Figure 4. Front panel showing delta, theta,alpha beta and gamma waveforms graphs and their FFT plots along with their mean, variance and standard deviation values

\section{E. Analysis}

Statistical Package for Social Sciences (SPSS) software tool was used for the statistical analysis. Initially, the power spectrum of mean EEG data was plotted using 25 line graphs (one graph for each subject) for investigating the changes in the signals due to meditation.

Further, entropies of all the five frequency bands were calculated in SPSS software. Since most of the data was not normally distributed, the non-parametric entropy tests were applied. The statistical analysis was undertaken to compare the both the result data of 25 subjects in terms of frequency bands (delta1, delta2, theta1, theta2, alpha1, alpha2, beta1, beta2, gamma1 and gamma2).
Here, delta1, theta1, alpha1, beta1 and gamma1 were brain waves recording during pre-test. On the other hand, delta2, theta2, alpha2, beta2 and gamma2 were brain waves recording during post-test. The entropies of all subjects were plotted using bar graphs. Fig. 4 shows the bar plot for all bands of one of the subject.

\section{RESULTS AND DISCUSSIONS}

The results revealed that during post test, there was a marked increase in activity within the frontal and occipital lobes of the brain. As the frontal areas are involved in focusing attention and advanced cognitive function. In past, researchers proved that when focusing attention on a task, the frontal areas of the brain become increasingly active. As a result of this particular type of meditation, with increase of frontal activity alpha wave tends to decrease. Whereas due to, increase in occipital activity there was rise in delta and theta waves.

From the observations, reduction in alpha rhythms indicates reduction in number of thoughts which signifies that mind is more regulated. Moreover, less thoughts means high focus, this improves the mental skills of persons to cope with stressful situations. On the other hand, rise in delta waves is helpful to achieve relaxed state and the person is less influenced by stress and performance level is also better. Furthermore, slight rise in theta wave activity in occipital region indicates the increases creativity, better coping abilities, more productivity and improvement in memory. In contrast, almost negligible differences in beta and gamma waves were observed.

Line graphs depicts that almost all the subjects have shown a noticeable variation in pre and post EEG data. The post graphs are more clear and smooth with sharp spikes. This indicates about the positive and constructive progress for brain. Line graph of one subject is shown in Fig. 5.

Also, marked improvements were noticed in not only the subject attention, but also in their working memory as their post-test scores were higher than pre-test scores.

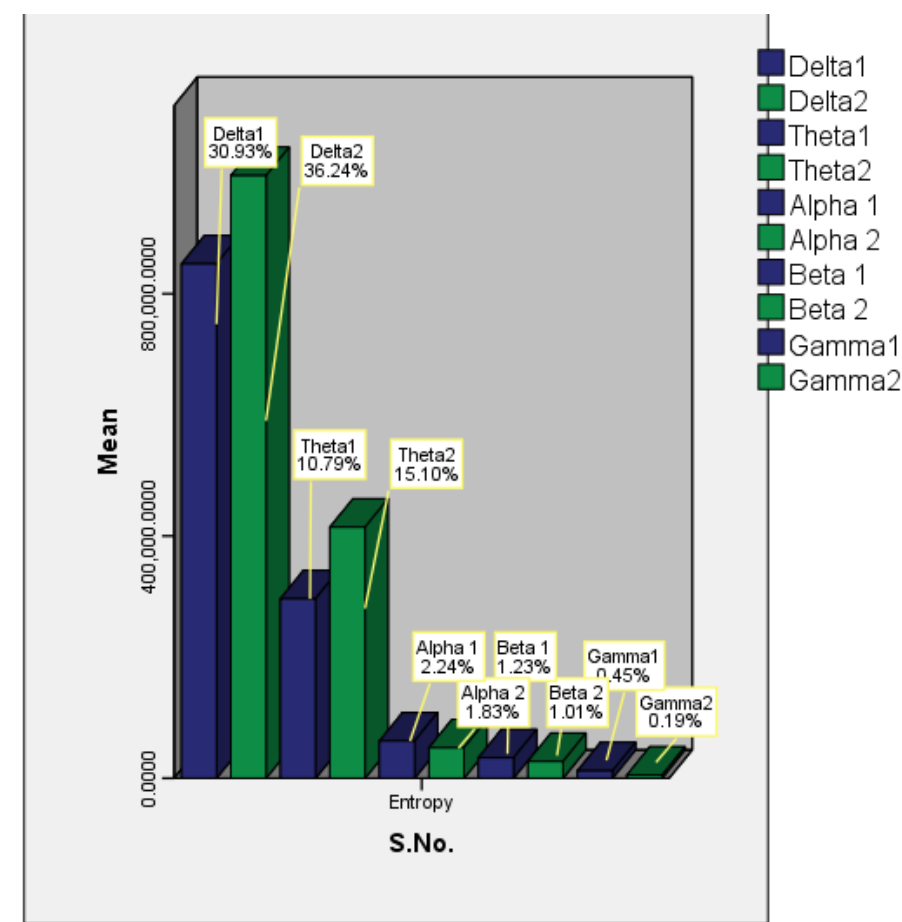

Figure 5. Entropy per each band for one of the subject 


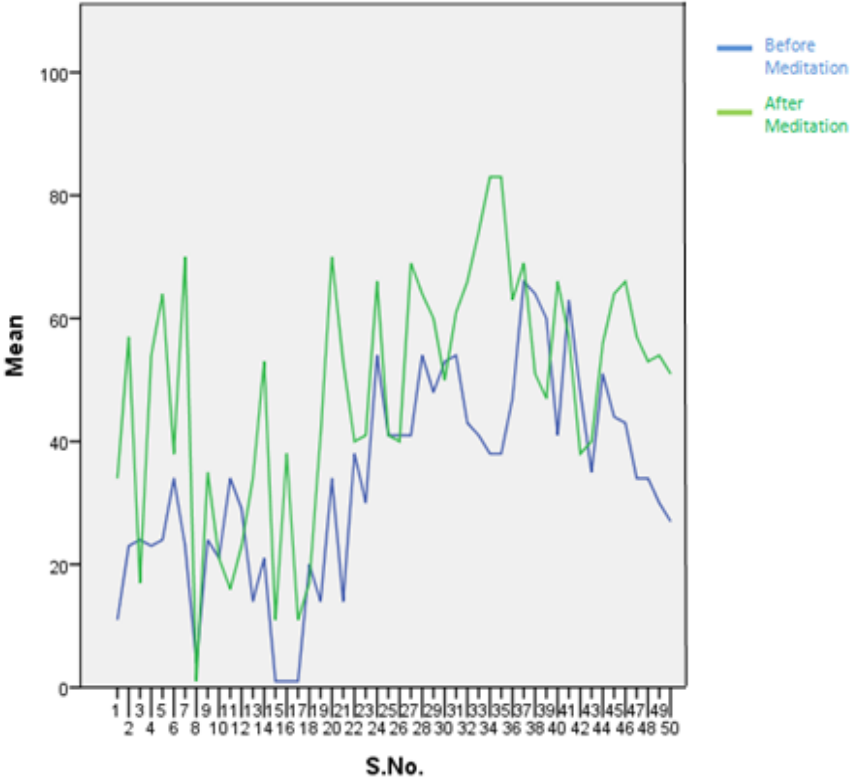

Figure 6. Line graph showing EEG graph for one of the subject

\section{CONCLUSION}

In recent years, meditations have come to be associated with thing like reduction in anxiety, better cognitive abilities, lower rate of depression, and greater degree of self control. In this paper, EEG (Electroencephalogram) signals of 25 subjects were recorded before and after a meditation intervention. From the recorded EEG signals, it was observed that after meditation sessions there was increase in delta and theta activity within the frontal and occipital lobes of the brain. In contrast, reduction in alpha wave activity and negligible changes in beta and gamma brainwaves were noticed. All the parameters included FFT, mean, variance and standard deviation, were considered to carry out the analysis in the LabVIEW (Laboratory Virtual Instrument Engineering Workbench) software. Statistical Package for Social Sciences (SPSS) software tool evaluated the entropies of the recorded parameters. This analysis is also compared with the existing paradigms for validation of the results. It was concluded that our approach was successful in predicting human behavior and conduct. The future scope of this research could be extended for diagnosis of patients suffering from anxiety and depression.

\section{REFERENCES}

[1] Hu, B., Peng, H., Zhao, Q., Hu, B., Majoe, D., Zheng, F., \& Moore, P. (2015). Signal Quality assessment model for wearable EEG sensor on prediction of mental stress. IEEE transactions on nanobioscience, 14(5), 553-561.

[2] Kumar, J. S., \& Bhuvaneswari, P. (2012). Analysis of Electroencephalography (EEG) Signals and Its Categorization-A Study. Procedia Engineering, 38, 2525-2536.

[3] Spencer, S. S. (1994). The relative contributions of MRI, SPECT, and PET imaging in epilepsy. Epilepsia, 35(s6).

[4] Menon, R. S., Ogawa, S., Kim, S. G., Ellermann, J. M., Merkle, H., Tank, D. W., \& Ugurbil, K. (1992). Functional Brain Mapping Using Magnetic Resonance Imaging: Signal Changes Accompanying Visual Stimulation. Investigative radiology, 27, S47-S53.

[5] Abdulkader, S. N., Atia, A., \& Mostafa, M. S. M. (2015). Brain computer interfacing: Applications and challenges. Egyptian Informatics Journal, 16(2), 213-230.

[6] Ames, F. R. (1971). " Self-induction" in photosensitive epilepsy. Brain: a journal of neurology.

[7] Trudeau, D. L. (2005). Applicability of brain wave biofeedback to substance use disorder in adolescents. Child and Adolescent Psychiatric Clinics of North America, 14(1), 125-136.

[8] Claassen, J., Hirsch, L. J., \& Mayer, S. A. (2003). Treatment of status epilepticus: a survey of neurologists. Journal of the neurological sciences, 211(1), 37-41.

[9] Fulpatil, P., \& Meshram, Y. (2014). Review on Analysis of EEG Signals with the Effect of Meditation. Int. Journal of Engineering Research and Applications June.

[10] Hall, M., Vasko, R., Buysse, D., Ombao, H., Chen, Q., Cashmere, J. D., ... \& Thayer, J. F. (2004). Acute stress affects heart rate variability during sleep. Psychosomatic medicine, 66(1), 56-62.

[11] Jacobs, G. D., \& Friedman, R. (2004). EEG spectral analysis of relaxation techniques. Applied psychophysiology and biofeedback, 29(4), 245-254.

[12] Takahashi, T., Murata, T., Hamada, T., Omori, M., Kosaka, H., Kikuchi, M., ... \& Wada, Y. (2005). Changes in EEG and autonomic nervous activity during meditation and their association with personality traits. International Journal of Psychophysiology, 55(2), 199-207.

[13] Travis, F., \& Wallace, R. K. (1999). Autonomic and EEG patterns during eyes-closed rest and transcendental meditation (TM) practice: the basis for a neural model of TM practice. Consciousness and cognition, 8(3),302-31. 